\title{
DETECCIÓN ESPECÍFICA DE TETRACICLINAS EN LECHE MEDIANTE LECTURAS FOTOMÉTRICAS DE UN BIOENSAYO QUE UTILIZA BACILLUS CEREUS
}

\author{
Nagel, O. G. ${ }^{1} ;$ Althaus, R. L. ${ }^{1}$ \& Molina, M. P. ${ }^{2}$
}

\begin{abstract}
RESUMEN
El objetivo de este trabajo fue determinar los límites de detección de 26 antimicrobianos utilizando las lecturas fotométricas de un bioensayo que utiliza Bacillus cereus. Para ello, se ensayaron 16 réplicas de 12 concentraciones utilizando muestras de leche procedentes de vacas individuales no tratadas ni medicamentadas. Los límites de detección del bioensayo se calcularon mediante el modelo de regresión logística. Las tetraciclinas detectan $60 \mu \mathrm{g}^{-1}$ de oxitetraciclina, $80 \mu \mathrm{g} \mathrm{l}^{-1} \mathrm{de}$ tetraciclina y $260 \mu \mathrm{g} \mathrm{l}^{-1}$ de clortetraciclina, valores similares al LMR $\left(100 \mu \mathrm{g} \mathrm{l}^{-1}\right)$. Este bioensayo no presenta especificidad cruzada con los antibióticos betalactámicos $\left(24800 \mu \mathrm{g}^{-1}\right.$ amoxicilina, 37500 $\mu \mathrm{g} \mathrm{l}^{-1}$ ampicilina, $10280 \mu \mathrm{g} \mathrm{l}^{-1}$ cloxacilina, $4500 \mu \mathrm{g} \mathrm{l}^{-1}$ oxacilina, $31400 \mu \mathrm{g}^{1^{-1}}$ penicilina $\mathrm{G}, 4330$ $\mu \mathrm{g} \mathrm{l^{-1 }}$ cefadroxilo, $3700 \mu \mathrm{g} \mathrm{l}^{-1}$ cefalexina, $7400 \mu \mathrm{g} \mathrm{l}^{-1}$ cefoperazone, $29200 \mu \mathrm{g} \mathrm{l}^{-1}$ ceftiofur ${ }^{\circledR}, 11260$ $\mu \mathrm{g}^{-1}$ cefuroxime), aminoglucósidos (2470 $\mu \mathrm{g} \mathrm{l}^{-1}$ gentamicina, $7000 \mu \mathrm{g}^{-1}$ kanamicina, $9950 \mu \mathrm{g} \mathrm{l}^{-1}$ neomicina, $8640 \mu \mathrm{g} \mathrm{l}^{-1}$ estreptomicina), macrólidos (1330 $\mu \mathrm{g} \mathrm{l}^{-1}$ eritromicina, $3470 \mu \mathrm{g} \mathrm{l}^{-1}$ lincomicina, $1170 \mu \mathrm{g}^{-1}$ tilosina), sulfamidas (65150 $\mu \mathrm{g}^{1^{-1}}$ sulfadiazina, $34160 \mu \mathrm{g}^{-1}$ sulfamerazina, $43500 \mu \mathrm{g}$ $\mathrm{l}^{-1}$ sulfametazina, $69800 \mu \mathrm{g} \mathrm{l}^{-1}$ sulfametoxazol, $52000 \mu \mathrm{g} \mathrm{l}^{-1}$ sulfatiazol $)$ y quinolonas $\left(1640 \mu \mathrm{g} \mathrm{l}^{-1}\right.$ ciprofloxacina, $1690 \mu \mathrm{g} \mathrm{l}^{-1}$ enrofloxacina, $1790 \mu \mathrm{g} \mathrm{l}^{-1}$ marbofloxacina, $4830 \mu \mathrm{g} \mathrm{l}^{-1}$ norfloxacina) por presentar límites de detección muy alejados de sus respectivos LMRs. Se concluye que el bioensayo resulta específico para la detección de residuos de tetraciclinas en leche. Además, a fin de evitar posibles errores debido a la interpretación de los resultados, se puede automatizar las lecturas mediante un lector de microplacas ELISA.
\end{abstract}

Palabras clave: antimicrobianos, Bacillus cereus, leche, método de inhibición microbiológica, detección fotométrica.

\footnotetext{
1.- Cátedra de Biofísica. Facultad de Ciencias Veterinarias (UNL). Kreder 2805. (3080) Esperanza, provincia de Santa Fe.

2.- Departamento de Ciencia Animal, Universidad Politécnica de Valencia. Camino de Vera 14, 46072, Valencia, España.

Manuscrito recibido el 20 de agosto de 2013 y aceptado para su publicación el 13 de diciembre de 2013.
} 


\section{SUMMARY}

\section{Specific detection of tetracycline in milk by photometric readings of a bioassay with Bacillus cereus.}

The aim of this work was to determine the detection limits of 26 antimicrobials using photometric readings of a bioassay that use Bacillus cereus. For this, 16 replicas were assayed using 12 concentrations of milk samples from untreated individual cows. The limits of detection of the bioassay were calculated by logistic regression model. Tetracyclines detect $60 \mathrm{mg} \mathrm{l}^{-1}$ of oxytetracycline, $80 \mathrm{mg} \mathrm{l}^{-1}$ of tetracycline and $260 \mathrm{mg} \mathrm{l}^{-1}$ of chlortetracycline, similar values to the MRL $\left(100 \mathrm{mg} \mathrm{l}^{-1}\right)$. This bioassay does not have cross-specificity with beta-lactam antibiotics (24800 $\mathrm{mg} \mathrm{l}^{-1}$ amoxicillin, $37500 \mathrm{mg}$ $\mathrm{l}^{-1}$ ampicillin, $10280 \mathrm{mg} \mathrm{l}^{-1}$ cloxacillin, $4500 \mathrm{mg} \mathrm{l}^{-1}$ oxacillin, $31400 \mathrm{mg} \mathrm{l}^{-1}$ penicillin $\mathrm{G}, 4330 \mathrm{mg} \mathrm{l}^{-1}$ cefadroxil, $3700 \mathrm{mg} \mathrm{l}^{-1}$ cephalexin, $7400 \mathrm{mg} \mathrm{l}^{-1}$ cefoperazone, $29200 \mathrm{mg} \mathrm{l}^{-1}$ ceftiofur ${ }^{\circledR}, 11260 \mathrm{mg}$ $1^{-1}$ cefuroxime), aminoglycosides (2470 mg 1-1 gentamycin, $7000 \mathrm{mg} \mathrm{1-1} \mathrm{kanamycin,} 9950 \mathrm{mg} \mathrm{l}^{-1}$ neomycin, $8640 \mathrm{mg} \mathrm{l}^{-1}$ streptomycin), macrolides (1330 $\mathrm{m} \mathrm{l}^{-1}$ erythromycin, $3470 \mathrm{mg} \mathrm{l}^{-1}$ lincomycin, $1170 \mathrm{mg} \mathrm{l}^{-1}$ tylosin), sulfonamides (65150 $\mathrm{mg} \mathrm{l}^{-1}$ sulfadiazine, $34160 \mathrm{~g} \mathrm{l}^{-1}$ sulfamerazine, $43500 \mathrm{mg}$ $\mathrm{l}^{-1}$ sulfamethazine, $69800 \mathrm{mg} \mathrm{l}^{-1}$ sulfamethoxazole, $52000 \mathrm{mg} \mathrm{l}^{-1}$ sulfathiazole) and quinolones (1640 $\mathrm{mg} \mathrm{l}^{-1}$ ciprofloxacin, $1690 \mathrm{mg} \mathrm{l}^{-1}$ enrofloxacin, $1790 \mathrm{mg} \mathrm{l}^{-1}$ marbofloxacin, $4830 \mathrm{mg} \mathrm{l}^{-1}$ norfloxacin) because their detection limit are greater than respective MRLs. It is concluded that, the bioassay is specific for detection of tetracycline residues in milk. In addition, to avoid possible errors due to the interpretation of the results, the readings can be automated through an ELISA microplate reader.

Key words: antimicrobial, Bacillus cereus, milk, microbial inhibitor test, photometric detection.

\section{INTRODUCCIÓN}

Las tetraciclinas (TCs) son antibióticos de amplio espectro que actúan inhibiendo la biosíntesis proteica mediante el bloqueo de la unión del ARN mensajero con la subunidad 30S del ribosoma bacteriano. Se utilizan con frecuencia en los tratamientos de infecciones bacterianas tales como mastitis, metritis y contra una amplia gama de patógenos, debido principalmente a su bajo costo y su relativa baja toxicidad (Chopra et al., 1992; Roberts, 1996).

Estas sustancias, al igual que otros antibióticos, pueden causar problemas asociados con síntomas alérgicos, desarrollo de resistencias a microorganismos (McManus, 1997; Demoly \& Romano, 2005). Además, pueden ocasionar pérdidas económicas a las industrias de productos lácteos (inhibición de los cultivos iniciadores utilizados en la fermentación de quesos y yogures) o en la aceptación final de estos productos debido a modificaciones en sus propiedades organolépticas (Perreten \& Teuber, 1995; Packham et al., 2001, Berruga et al., 2009).

Por ello, un monitoreo continuo de los residuos de antibióticos debe realizarse con el propósito de garantizar la seguridad en la cadena alimentaria. Por este motivo, la Comisión de Regulación de la Comunidad Europea (2010) determina los Límites Máximos Residuos (LMRs) para los diferentes productos veterinarios que pueden estar presentes en los alimentos, incluida la leche. En el caso concreto de las tetraciclinas, la legislación establece únicamente un nivel de $100 \mu \mathrm{g}^{-1}$ para residuos de tetraciclina, 